\title{
BackPocketDriver - A Mobile App to Enhance Safe Driving for Youth
}

\author{
Catherine Shanly, Michael Ieti \\ Department of Electrical and Computer Engineering \\ The University of Auckland, New Zealand \\ \{csha289,miet001\}@aucklanduni.ac.nz
}

\author{
Ian Warren and Jing Sun \\ Department of Computer Science \\ The University of Auckland, New Zealand \\ \{i-warren,j.sun\}@cs.auckland.ac.nz
}

\begin{abstract}
Young drivers are one of the highest risk groups for being involved in car accidents. BackPocketDriver (BPD) is an Android application that aims at encouraging young drivers to adopt and hone safe driving skills. Smartphone sensors are used to monitor driver behaviour, including speed, turning, acceleration and braking. The journey data is analysed for unsafe behaviour with user feedback, which includes journey review, positive reinforcement using textual messages, goal setting and points scoring. To achieve this, behavioural change techniques were studied in relation to gamification, and several features were implemented into BackPocketDriver including achievements, leader board, quizzes, friends system, etc. Evaluations in terms of functional comparisons with related tools were conducted to measure the advantages of the proposed solution. The BPD app provides effective improvements to youth driving.
\end{abstract}

\section{INTRODUCTION}

Youth drivers are one of the most at-risk groups in New Zealand with regards to car accidents. From 2013-2015, drivers from 15-24 accounted for only $13 \%$ of all licensed drivers, but were involved in $24 \%$ of all minor crashes, $23 \%$ of all major crashes, and $19 \%$ of all fatal crashes [1]. These crashes resulted in more than 200 deaths in those two years.

Further investigation into the causes of these crashes resulted in a number of primary factors being identified. The main factors were alcohol/drug consumption, losing control, and speed. More than half $(53 \%)$ of all fatal crashes had alcohol, drug use, or speeding identified as being contributing factors. Young drivers are more than twice as likely to have speed or alcohol as a crash factor compared to drivers over the age of 25 [1]. In a report by Transport Engineering Research New Zealand (TERNZ), it is stated that most fatal crashes occur at speeds over $60 \mathrm{~km} / \mathrm{hr}$ [2]. These statistics highlight points where action can be taken to reduce deaths - reducing speed and reinforcing safe driving behaviours and habits may be the key to minimising drivingrelated injuries and deaths in New Zealand.

Avoidable crashes also create a large amount of economic, financial, and intangible cost for society. These costs are largely attributed to reduced quality of life for victims, fatalities, loss of productivity, as well as medical and legal fees, totaling \$3.79 billion in the year 2015. Vehicle damage only accounts for around $5 \%$ of this cost [3].

DOI reference number: 10.18293/SEKE2018-011
The ubiquity of smartphones in all demographics opens a channel for engagement with drivers of all ages. Smartphones have reached $70 \%$ market penetration in New Zealand, with $91 \%$ of 18-34 year olds owning at least one smartphone [4-7]. In order to focus efforts towards younger drivers, the use of a smartphone app can be seen as an appropriate measure to analyse and correct unsafe driving behaviours [8].

BackPocketDriver (BPD) is an Android application that aims at encouraging young drivers to adopt and hone safe driving skills. Smartphone sensors are used to track a user's driving, such as speed, turning, acceleration, braking, etc. Behavioural Change Techniques (BCT) $[3,4]$ and gamification ideas $[9,10]$ were adopted in the development of the tool. Users can set their own goals to improve the smoothness of their driving and to reduce their speeding. The data is then analysed for unsafe behaviour through user feedback. A journey summary is provided after a trip, detailing the route taken, the user's current goals, and if they have met them. In this summary, specific feedback is also given to the user. An additional feature of BackPocketDriver is a daily messaging system gives the user safe driving tips or instructions daily.

The rest of the paper is organised as follows. In Section II, we present the development of the BackPocketDriver app. Section III presents the evaluation of the tool, including the feature comparison to existing safe driving applications. Finally, Section VI concludes the paper and outline the future work.

\section{DESIGN AND IMPLEMENTATION}

\section{A. Overall Technologies}

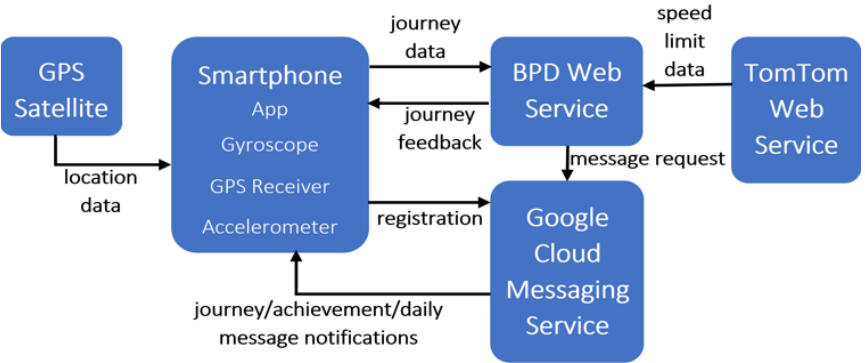

Fig. 1. BackPocketDriver component diagram

Fig. 1 shows the components of BackPocketDriver and its overall architecture. The user's smartphone uses the GPS, accelerometer, and gyroscope to calculate journey data. This is 
then sent to the BackPocketDriver web service for processing before journey feedback is sent back to the device. A messaging service is also used for registration purposes and to send daily messages and notifications to the user.

We integrated several technologies for the development of the app and server for BackPocketDriver. The app is an Android smartphone app and the server code was implemented in Java programming language. The MySQL relational database management system was used for the server while SQLite was used for the app. Additionally, the GreenDAO generator was used in the app to map Java objects to the relational database. We used Apache Tomcat, an open-source servlet container that provides an HTTP web server environment. We used Google Cloud Platform to host the application.

\section{B. Key Features}

Fig. 2 shows the Home screen that the user first sees when they open BackPocketDriver. Users can start and stop recording their journey on this screen by pressing the car icon. The username of the user is displayed which allows the user to identify themselves and more easily compare themselves with others.

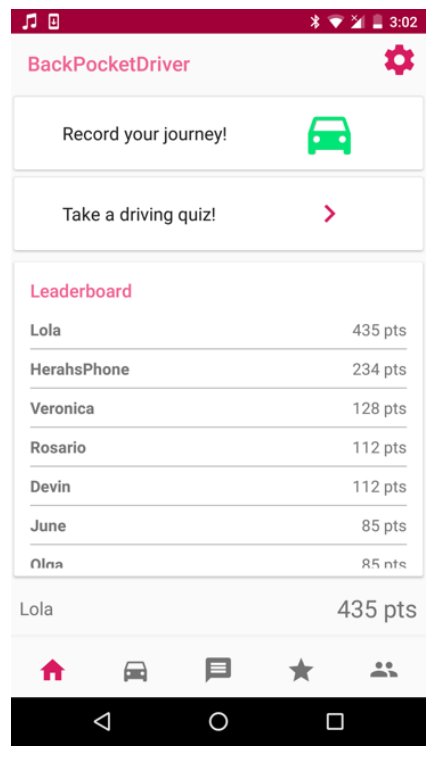

Fig. 3. Home screen implementation

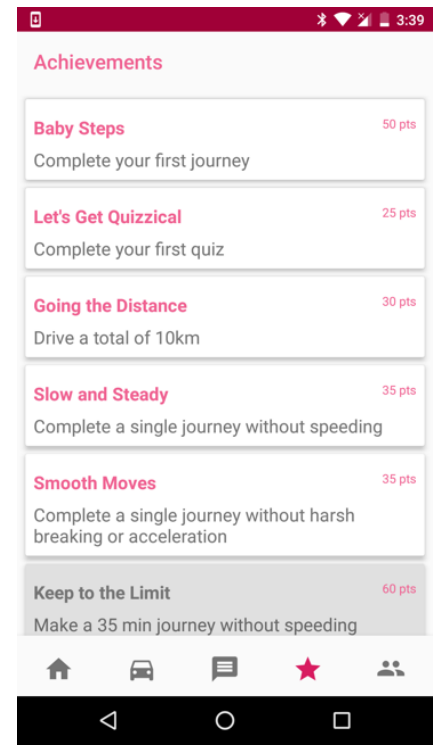

Fig. 3. Achievements implementation
The user can click on the gear icon to access user preferences. This gives the user control over their use of the app, for example, in our implementation users can choose to appear on the global leaderboard. The use of preferences can promote feelings of autonomy, which increases motivation [15], but is also important because BackPocketDriver contains potentially sensitive information about users.

On the Home screen the user can also see how many points they have earned. This is immediately available for the user to see and links with the BCT [12] of feedback on behaviour as users will see an increase in their score as soon as they perform a good behaviour, such as recording a journey without harsh braking.
The leaderboard has also been implemented on this screen. The leaderboard shows the top-ten users and ranks them against the number of points they have earned (users can earn points by recording journeys, completing quizzes, and obtaining achievements). Users are motivated to perform point earning activities when they compare their score against others and are motivated to continue performing them if they see themselves as a role model on the top of the leaderboard [11].

Fig. 3 shows the Achievements screen where users can obtain achievements by carrying out certain tasks, for example, the "Baby Steps" achievement requires that the user complete a journey for the first time. Achievements are motivating as users can set goals for themselves to obtain a specific achievement and they also visually show (achievement items are no longer greyed out) a sense of progress for the user as they obtain more achievements [14].

We have implemented achievements so that users can earn points when they complete a specific task. Each achievement has a number of points associated with it making the use of points more meaningful as users know exactly that they need to do, for example driving a total of $10 \mathrm{~km}$, to earn points.

There are two different types of achievements implemented in BackPocketDriver. The "Let's Get Quizzical" achievement, for example, directs users towards the quiz functionality in the app. This can help direct users to functionality that they may not be aware of. Alternatively, the "Smooth Moves" achievement motivates the users to display good driving behaviours (completing a journey without harsh braking or acceleration) [13]. This links with the BCT of instruction on how to perform a behaviour as it explicitly shows the user what is considered good driving behaviours [16].

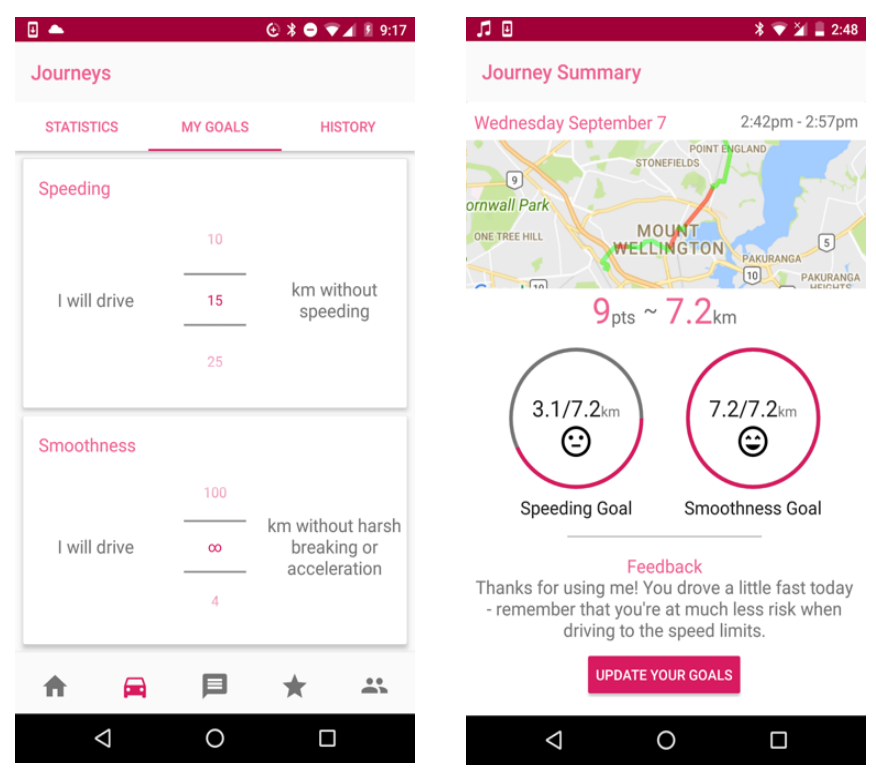

Fig. 4. My Goals screen

Fig. 5. Journey Summary screen

Fig. 4 and 5 show the screens for the redefining trip goals, reviewing trip goals after a journey, and redesigning journey feedback requirements. Fig. 4, the My Goals screen, allows the user to set themselves a speeding goal and a smoothness goal for each journey. In our implementation, the goals are clearly 
TABLE I

SUMMARY OF FEATURES

\begin{tabular}{|c|c|c|c|c|c|c|}
\hline \multirow[b]{2}{*}{ BCTs } & \multicolumn{6}{|c|}{ Feature } \\
\hline & Points & Leaderboard & Achievements & Quizzes & Friends & (Redefined) Trip Goals \\
\hline Feedback on behaviour & $\mathrm{X}$ & & $\mathrm{X}$ & & & $\mathrm{X}$ \\
\hline Social comparison & & $\mathrm{X}$ & $\mathrm{X}$ & & $\mathrm{X}$ & \\
\hline Goal-setting & & $\mathrm{X}$ & $\mathrm{X}$ & & & $X$ \\
\hline Identification of self as role-model & & $\mathrm{X}$ & & & & \\
\hline Instruction on how to perform a behaviour & & & $\mathrm{X}$ & $\mathrm{X}$ & & \\
\hline Discrepancy between current behaviour and goal & & & & & & $\mathrm{X}$ \\
\hline Review behaviour and goals & & & & & & $\mathrm{X}$ \\
\hline
\end{tabular}

defined, for example, on the My Goals screen the speeding goal is "I will drive $15 \mathrm{~km}$ without speeding." The user can scroll through a list of available numbers to change the goal depending on their ability. The " $\infty$ " indicates that the user, for example, will not brake or accelerate harshly for the whole duration of their journey. If the length of the user's trip is less than their current goal, then the user must also drive the whole trip perfectly.

Fig. 5 shows the Journey Summary screen which has been updated from the original app to reflect the changes in the goals. There is now a circular progress bar that shows the user progress towards their goal as well as the exact distance they have driven without speeding and the distance they have driven smoothly. These two features show the discrepancy between the user's behaviour and their goal, another BCT.

On the Journey Summary screen, we have implemented a button that prompts the user to update their goals after they have seen their journey feedback and if they have met their goals. This leads the user back to the My Goals screen, allowing them to review their goals. Redefining the journey goals involved many changes across the app and server code. Journeys had to be processed differently so that the distance speeding and driving harshly was calculated, stored, and sent to the app as part of a journey summary object (previously only the proportion of the distance speeding was stored).

\section{C. $\quad$ Summary of Implemented Features}

Table I shows a summary of the features implemented during the timeline of this project and the BCTs they are associated with. Additionally, there are other links between features. For example, users are ranked on the leaderboard based on how many points they have, which they can earn by recording journeys, achievements, and completing quizzes. Achievement activities correspond to several other functionalities of the app and support the behavioural change ideas, e.g., the achievement for appearing on the leaderboard is named "Role Model". The friends system also allows users to view the achievements of their friends. These links all show how the implemented features are designed to support and reinforce each other.

\section{EVALUATION}

\section{A. Performance Measurements}

The performance of the server was briefly analysed using loader.io, a website that can stress test web applications with multiple concurrent connections [17]. Our tests retrieved the top ten users on the leaderboard where each test had, over a 1 minute period, an increasing amount of client requests.

There were no errors up to 500 client requests over a minute. This test had an average response time of $70 \mathrm{~ms}$. 1000 clients had a $0.04 \%$ error rate and an average response time of $133 \mathrm{~ms}$. For 5000 clients, there was an $0.82 \%$ error rate with an average response time of $156 \mathrm{~ms}$. 10,000 clients over a 1 minute period had a $25.21 \%$ error rate with an average response time of $1224 \mathrm{~ms}$. All errors were timeouts (set at 10 seconds).

This shows that as the number of clients increase the longer the response time is and the more errors there are due to timeouts. Our web server has therefore limited scalability. This problem needs to be addressed before BackPocketDriver is made available to more users.

\section{B. Comparisons of BackPocketDriver to Other Safe Driving Applications}

Table II shows a selection of safe driving applications, available on the Google Play Store, and their features (based on the app description). The most similar app to BackPocketDriver is the TOWER Insurance SmartDriver app [18], however, as this has been created by an insurance company users may not be motivated to use the app if they initially identify as a poor driver.

Some gamification features, such as points, appear to be popular across some of the other apps. Other features that BackPocketDriver does not include are a safe driving mode where the app discourages the user from handling their phone while driving. Instead, the app may perform tasks on behalf of the user using voice commands.

The data demonstrates that there is a gap in safe driving apps that BackPocketDriver has the potential to fill. The proposed solution uses a range of gamification features and uses a social network (friends system) which is not otherwise common. In the future, BackPocketDriver could support some of the other features present in the other apps to appeal to a wider audience.

\section{CONCLUSIONS}

The relationship between BCTs and gamification was examined and a clear link was found between these two concepts. The results of this study were applied to BackPocketDriver, a smartphone application created for encouraging safe driving in youth drivers. Limitations of the existing app were addressed by extending existing features and by implementing the following game elements: achievements, leaderboards, and quizzes. Additionally, a friends system was 
TABLE II

COMPARING BACKPocketDriver to OTHER SAFE Driving APPS

\begin{tabular}{|c|c|c|c|c|c|c|}
\hline \multirow[b]{2}{*}{ Feature } & \multicolumn{6}{|c|}{ Application } \\
\hline & BackPocketDriver & $\begin{array}{l}\text { TOWER Insurance } \\
\text { SmartDriver [18] }\end{array}$ & $\begin{array}{l}\text { Dash - Drive } \\
\text { Smart [19] }\end{array}$ & $\begin{array}{c}\text { Drive Safe } \\
{[20]}\end{array}$ & $\begin{array}{c}\text { Safe Driving App } \\
{[21]}\end{array}$ & $\begin{array}{c}\text { EROAD Driver } \\
{[22]}\end{array}$ \\
\hline Journey feedback & $\mathrm{X}$ & $\begin{array}{l}\mathrm{X} \\
\end{array}$ & $\mathrm{X}$ & & $\mathrm{X}$ & \\
\hline Goals & $\mathrm{X}$ & & & & & \\
\hline Statistics & $\mathrm{X}$ & X & & $\mathrm{X}$ & $X$ & \\
\hline Daily messages & $\mathrm{X}$ & & & & & \\
\hline Points/Score & $\mathrm{X}$ & $\mathrm{X}$ & $X$ & & $\mathrm{X}$ & \\
\hline Leaderboard & $\mathrm{X}$ & $\mathrm{X}$ & $\mathrm{X}$ & & & \\
\hline Quizzes & $\mathrm{X}$ & & & & & \\
\hline Achievements & $\mathrm{X}$ & $\mathrm{X}$ & & & & \\
\hline Rewards & & $\mathrm{X}$ & & & & \\
\hline Social network & $\mathrm{X}$ & $\mathrm{X}$ & & & & \\
\hline Logging driving & & & & & & $\mathrm{X}$ \\
\hline $\begin{array}{c}\text { Vehicle inspection } \\
\text { details }\end{array}$ & & & & & & $\mathrm{X}$ \\
\hline Driving tips & & & & & & $\mathrm{X}$ \\
\hline Safe driving mode & & & $\mathrm{X}$ & X & & \\
\hline
\end{tabular}

implemented. These features were chosen based on their motivational effectiveness and their relevance to BCTs. They were evaluated by conducting a performance and comparative study to related tools. The results found that proposed solution provides effective means to improve youth driving.

In the future, the validity of the app needs to be evaluated by carrying out a long-term study of users recording their driving with the app. During this study the motivational effectiveness of features could be evaluated by recording how often the user visits different functionality as well as seeing if their driving performance improves over time.

\section{REFERENCES}

[1] Young Drivers I NZ Transport Agency. Available: http://www.nzta.govt.nz/safety/driving-safely/young-drivers/.

[2] Young drivers crash facts I Ministry of Transport. Available: http://www.transport.govt.nz/research/crashfacts/youngdriverscrashfacts

[3] S. Michie et al, "The behavior change technique taxonomy (v1) of 93 hierarchically clustered techniques: building an international consensus for the reporting of behavior change interventions," Annals of Behavioral Medicine, vol. 46, (1), pp. 81-95, 2013.

[4] C. Abraham and S. Michie, "A taxonomy of behavior change techniques used in interventions." Health Psychology, vol. 27, (3), pp. 379, 2008.

[5] A. Direito et al, "Do physical activity and dietary smartphone applications incorporate evidence-based behaviour change techniques?" BMC Public Health, vol. 14, (1), pp. 646, 2014.

[6] C. C. Quinn et al, "Cluster-randomized trial of a mobile phone personalized behavioral intervention for blood glucose control. Diabetes Care 2011; 34: 1934-1942," Diabetes Care, vol. 36, (11), pp. 3850, 2013.

[7] F. Fylan and S. Stradling, "Behavioural Change Techniques used in road safety interventions for young people," Revue Europenne De Psychologie Applique/European Review of Applied Psychology, vol. 64, (3), pp. 123129, 2014.

[8] S. Deterding et al, "From game design elements to gamefulness: Defining gamification," in Proceedings of the 15th International Academic MindTrek Conference: Envisioning Future Media Environments, 2011, pp. 9-15.
[9] M. Sailer et al, "How gamification motivates: An experimental study of the effects of specific game design elements on psychological need satisfaction," Comput. Hum. Behav., vol. 69, pp. 371-380, 2017.

[10] J. Hamari, J. Koivisto and H. Sarsa, "Does gamification work?--a literature review of empirical studies on gamification," in System Sciences (HICSS), 2014 47th Hawaii International Conference On, 2014, pp. 3025-3034.

[11] R. N. Landers, K. N. Bauer and R. C. Callan, "Gamification of task performance with leaderboards: A goal setting experiment," Comput. Hum. Behav., 2015.

[12] J. Hamari, "Do badges increase user activity? A field experiment on the effects of gamification," Comput. Hum. Behav., 2015.

[13] C. Cheong, F. Cheong and J. Filippou, "Quick quiz: A gamified approach for enhancing learning." in Pacis, 2013, pp. 206.

[14] E. A. Edwards et al, "Gamification for health promotion: systematic review of behaviour change techniques in smartphone apps," BMJ Open, vol. 6, (10), pp. e012447, 2016.

[15] R. M. Ryan and E. L. Deci, "Self-determination theory and the facilitation of intrinsic motivation, social development, and well-being." Am. Psychol., vol. 55, (1), pp. 68, 2000.

[16] L. de-Marcos, E. Garcia-Lopez and A. Garcia-Cabot, "On the effectiveness of game-like and social approaches in learning: Comparing educational gaming, gamification \& social networking," Comput. Educ., vol. 95, pp. 99-113, 2016.

[17] Application Load Testing Tools for API Endpoints with loader.io. Available: https://loader.io/.

[18] SmartDriver - Android Apps on Google Play. Available: https://play.google.com/store/apps/details?id=com.tower.smartdriver.

[19] Dash - Drive Smart - Android Apps on Google Play. Available: https://play.google.com/store/apps/details?id=com.dashlabs.dash.android

[20] Drive Safe - Android Apps on Google Play. Available: https://play.google.com/store/apps/details?id=westport.andrewirwin.com drivesafe.

[21] Safe Driving App - Android Apps on Google Play. Available: https://play.google.com/store/apps/details?id=com.risktechnology .ingdir ect.

[22] EROAD Driver - Android Apps on Google Play. Available: https://play.google.com/store/apps/details?id=com.eroad.driver. 